\title{
FUNCTIONS OF VANISHING MEAN OSCILLATION ASSOCIATED TO NON-NEGATIVE SELF-ADJOINT OPERATORS SATISFYING DAVIES-GAFFNEY ESTIMATES
}

\author{
THE ANH BuI
}

(Received October 26, 2011, revised May 28, 2013)

\begin{abstract}
Let $L$ be a nonnegative self-adjoint operator satisfying Davies-Gaffney estimates on $L^{2}(X)$, where $X$ is a metric space. In this paper, we introduce and develop a new function space $\operatorname{VMO}_{L}(X)$ of vanishing mean oscillation type associated to $L$. We then prove that the dual of $\mathrm{VMO}_{L}(X)$ is the Hardy space $H_{L}(X)$ which was investigated in [18]. Some characterizations of $\mathrm{VMO}_{L}(X)$ are also established.
\end{abstract}

1. Introduction. A locally integrable function $f$ defined on $\boldsymbol{R}^{n}$ is said to be in $\operatorname{BMO}\left(\boldsymbol{R}^{n}\right)$, the space of functions of bounded mean oscillation, if

$$
\|f\|_{\mathrm{BMO}}=\sup _{B} \frac{1}{|B|} \int_{B}\left|f(y)-f_{B}\right| d y<\infty,
$$

where the supremum is taken over all balls $B$ in $\boldsymbol{R}^{n}$, and $f_{B}$ stands for the mean of $f$ over $B$, i.e.,

(see [21]).

$$
f_{B}=\frac{1}{|B|} \int_{B} f(y) d y
$$

In [23], Sarason introduced the space of functions of vanishing mean oscillation, that is, the set of all functions of $\operatorname{BMO}\left(\boldsymbol{R}^{n}\right)$ satisfying the limiting condition

$$
\lim _{a \rightarrow 0}\left(\sup _{B: r_{B} \leq a} \frac{1}{|B|} \int_{B}\left|f(y)-f_{B}\right| d y\right)=0,
$$

which is denoted by $\operatorname{VMO}\left(\boldsymbol{R}^{n}\right)$. We endow $\operatorname{VMO}\left(\boldsymbol{R}^{n}\right)$ with the norm of $\operatorname{BMO}\left(\boldsymbol{R}^{n}\right)$.

In 1972, Fefferman and Stein [15] showed that $\operatorname{BMO}\left(\boldsymbol{R}^{n}\right)$ is the dual of the Hardy space $H^{1}\left(\boldsymbol{R}^{n}\right)$. Coifman and Weiss [11] then introduced a modified version of $\operatorname{VMO}\left(\boldsymbol{R}^{n}\right)$, denoted by $\operatorname{CMO}\left(\boldsymbol{R}^{n}\right)$, the closure of the space $C_{0}\left(\boldsymbol{R}^{n}\right)$ with respect to the BMO norm, where $C_{0}\left(\boldsymbol{R}^{n}\right)$ is the spaces of continuous functions with compact support. They proved that the space $H^{1}\left(\boldsymbol{R}^{n}\right)$ is the dual of $\operatorname{CMO}\left(\boldsymbol{R}^{n}\right)$.

In recent years, the study of Hardy spaces and BMO spaces associated to operators has attracted a lot of attentions (see for example [2, 4, 5, 13, 14, 17, 18, 19] and their references).

2010 Mathematics Subject Classification. Primary 42B20; Secondary 42B25.

Key words and phrases. Non-negative self-adjoint operator, Hardy space , BMO, VMO, space of homogeneous type.

The Anh Bui was supported by a Macquarie University scholarship. 
In [2], the authors studied the Hardy spaces associated to an operator whose heat kernel satisfies a pointwise Poisson upper bound. Later, the BMO spaces associated to such an $L$ were introduced in [13, 14]. Recently, Auscher, McIntosh and Russ treated the Hardy space $H^{1}$ associated to Hodge Laplacian on a Riemannian manifold (see [4]). Meanwhile, Hofmann and Mayboroda introduced the Hardy spaces associated to a second order divergence form elliptic operator $L$ on $\boldsymbol{R}^{n}$ with complex coefficients (see [17]). Then Hofmann et al. [18] introduced new Hardy spaces $H_{L}^{p}, 1 \leq p<\infty$, on a metric space $X$ associated to a non-negative selfadjoint operator $L$ satisfying Davies-Gaffney estimates. In the same setting as in [18], the authors in [19] introduced the Orlicz-Hardy spaces associated to $L$, a generalization of the Hardy space $H_{L}^{1}$ introduced in [18].

Continuing the line of study in $[2,13,17]$, the authors in $[12,20]$ introduced and developed a new function space $\mathrm{VMO}_{L}(X)$ of vanishing mean oscillation associated to an operator $L$ whose heat kernel satisfies a pointwise Poisson upper bound or a second order divergence form elliptic operator $L$ on $\boldsymbol{R}^{n}$ with complex coefficients, respectively. Motivated by these previous works, this paper is devoted to studying the new function spaces $\operatorname{VMO}_{L}(X)$ of vanishing mean oscillation associated to a non-negative self-adjoint operator satisfying so called Davies-Gaffney estimates. Compared with the work of [12], we do not require the pointwise estimate of the heat kernel. This is replaced by weaker estimates Davies-Gaffney estimates. This kind of estimates is common in the works of $[17,18,19,20]$. Let us remind you that the generalized vanishing mean oscillation spaces were investigated by R. Jiang and D. Yang [20] in Euclidean space $\boldsymbol{R}^{n}$ setting. Therefore, in some sense, the work in this paper can be considered to be an extension of those in [20].

The organization of this paper is as follows. In Section 2, we give some preliminaries. An important covering lemma (Lemma 2.1) is addressed in this section. In Section 3, we introduce the space $\mathrm{VMO}_{L}(X)$ and then establishes some characterizations of $\operatorname{VMO}_{L}(X)$. The main result of this paper is given in Section 4. It is shown that the dual of the space $\mathrm{VMO}_{L}(X)$ is the Hardy space $H_{L}(X)$ introduced in [18].

Throughout the paper, we always use $C$ and $c$ to denote positive constants that are independent of the main parameters involved but whose values may differ from line to line.

2. Notations and preliminaries. Let $X$ be a metric space with a distance $d$ and a nonnegative, doubling, Borel measure $\mu$ on $X$. Throughout this paper, we assume that $\mu(X)=\infty$. Denote by $B(x, r)$ the open ball of radius $r>0$ and center $x \in X$, and by $V(x, r)$ its measure $\mu(B(x, r))$. The doubling property of $\mu$ provides that there exists a constant $C_{1}>0$ so that

$$
V(x, 2 r) \leq C_{1} V(x, r)
$$

for all $x \in X$ and $r>0$. Notice that the doubling property (1) implies that there exists positive constants $n$ and $C_{2}$ so that

$$
V(x, \lambda r) \leq C_{2} \lambda^{n} V(x, r)
$$


for some positive constant $n$ uniformly for all $\lambda \geq 1, x \in X$ and $r>0$. There also exist constants $0 \leq N \leq n$ and $C_{3}$ such that

$$
V(x, r) \leq C_{3}\left(1+\frac{d(x, y)}{r}\right)^{N} V(y, r),
$$

uniformly for all $x, y \in X$ and $r>0$.

To simplify notation, we will often just use $B$ for $B\left(x_{B}, r_{B}\right)$. Given $\lambda>0$, we will write $\lambda B$ for the $\lambda$-dilated ball, which is the ball with the same center as $B$ and with radius $r_{\lambda B}=\lambda r_{B}$. For each ball $B \subset X$ we set

$$
S_{0}(B)=B \quad \text { and } \quad S_{j}(B)=2^{j} B \backslash 2^{j-1} B \quad \text { for } j \in N .
$$

The following covering lemma plays a key role in sequel.

LEMMA 2.1. Let $l$ and $r$ be two positive constants. For any ball $B\left(x_{B}, l r\right)$ in $X$, there exists a set of balls $\left\{B\left(x_{k_{1}}, r\right), \ldots, B\left(x_{k_{N_{k}}}, r\right)\right\}$ such that

(a) $B\left(x_{B}, l r\right) \subset \cup_{j=1}^{N_{k}} B\left(x_{k_{j}}, r\right)$;

(b) $N_{k} \leq C l^{n}$;

(c) $\sum_{j=1}^{N_{k}} \chi_{B\left(x_{k_{j}}, r\right)} \leq C$, where $C$ is independent of $l$ and $r$.

Proof. We consider the case when $l \geq 1$. Otherwise, there is nothing to prove. By Vitali covering lemma, we can pick from the family of balls $\left\{B\left(x, \frac{r}{3}\right) ; x \in B\left(x_{B}, l r\right)\right\}$ a disjoint family $\left\{B\left(x_{k_{1}}, \frac{r}{3}\right), \ldots, B\left(x_{k_{N_{k}}}, \frac{r}{3}\right)\right\}$ such that $B\left(x_{B}, l r\right) \subset \cup_{j=1}^{N_{k}} B\left(x_{k_{j}}, r\right)$. Obviously, $B\left(x_{k_{j}}, \frac{r}{3}\right) \subset B\left(x_{B}, 2 l r\right)$ and $B\left(x_{B}, l r\right) \subset B\left(x_{k_{j}}, 6 l \frac{r}{3}\right)$ for all $j=1, \ldots, N_{k}$. This together with (2) gives

$$
\begin{aligned}
V\left(x_{B}, l r\right) & \geq C_{1} V\left(x_{B}, 2 l r\right) \geq C \sum_{j=1}^{N_{k}} V\left(x_{k_{j}}, \frac{r}{3}\right) \\
& \geq C \sum_{j=1}^{N_{k}}(6 l)^{-n} V\left(x_{k_{j}}, 6 l \frac{r}{3}\right) \geq C \sum_{j=1}^{N_{k}} l^{-n} V\left(x_{B}, l r\right)=C N_{k} l^{-n} V\left(x_{B}, l r\right) .
\end{aligned}
$$

This implies $(b)$.

For any $x \in X$, set $I_{x}=\left\{i ; x \in B\left(x_{i}, r\right), i \in\left\{k_{1}, \ldots, k_{N_{k}}\right\}\right\}$. Then, $\cup_{i \in I_{x}} B\left(x_{i}, \frac{r}{3}\right) \subset$ $\cup_{i \in I_{x}} B\left(x_{i}, r\right) \subset B(x, 2 r)$. This implies

$$
\begin{aligned}
V(x, r) & \geq C V(x, 2 r) \geq \sum_{i \in I_{x}} V\left(x_{i}, \frac{r}{3}\right) \\
& \geq C \sum_{i \in I_{x}} V\left(x_{i}, 12 \frac{r}{3}\right) \geq\left|I_{x}\right| V(x, r) .
\end{aligned}
$$

It therefore follows that $\sum_{j=1}^{N_{k}} \chi_{B\left(x_{k_{j}}, r\right)} \leq C$, where $C$ is independent of $l$ and $r$. 
REMARK 2.2. It is easy to see that

$$
\max \left\{\frac{V\left(x_{k_{j}}, r\right)}{V\left(x_{B}, l r\right)}, \frac{V\left(x_{B}, l r\right)}{V\left(x_{k_{j}}, r\right)}\right\} \leq C(1+l)^{N} .
$$

2.1. Assumptions. The following will be assumed throughout the paper unless otherwise specified:

(H1) $L$ is a non-negative self-adjoint operator on $L^{2}(X)$.

(H2) The operator $L$ generates an analytic semigroup $\left\{e^{-t L}\right\}_{t>0}$ which satisfies the Davies-Gaffney estimate, i.e., there exist positive constants $C$ and $c$ such that for all closed sets $E$ and $F$ in $X, t \in(0, \infty)$ and $f \in L^{2}(X)$ supported in $E$,

$$
\left\|e^{-t L} f\right\|_{L^{2}(F)} \leq C \exp \left\{-\frac{d(E, F)^{2}}{c t}\right\}\|f\|_{L^{2}(E)},
$$

where $d(E, F)$ is the distance between the sets $E$ and $F$.

LEMMA 2.3 ([16]). If two families of operators, $\left\{S_{t}\right\}_{t>0}$ and $\left\{T_{t}\right\}_{t>0}$ satisfy the Davies-Gaffney estimate (4). Then there exist two constants $C \geq 0$ and $c>0$ such that, for every $t>0$, every closed subsets $E$ and $F$ of $X$ and every function $f$ supported in $E$,

$$
\left\|S_{s} T_{t} f\right\|_{L^{2}(F)} \leq C \exp \left\{-\frac{d(E, F)^{2}}{c \max \{s, t\}}\right\}\|f\|_{L^{2}(E)} .
$$

Examples of operators satisfying the assumptions (H1) and (H2) include degenerate Schrödinger operators with nonnegative potential, Schrödinger operators with nonnegative potential and magnetic field and Laplace-Beltrami operators on all complete Riemannian manifolds (see $[1,3,8]$ ).

Proposition 2.4 ([18]). For every $K \in N$, the family of operators $\left\{(t L)^{k} e^{-t L}\right\}_{t>0}$ satisfies the Davies-Gaffney estimate (4).

The proof is similar to one in [18] and hence we omit it here.

3. The spaces $\operatorname{VMO}_{L}(X)$.

3.1. Hardy spaces associated to operators. The theory of Hardy spaces associated to non-negative self-adjoint operators satisfying Davies-Gaffney estimates was developed recently by Hofmann et. al. [18]. Here, we use the definitions and characterizations of Hardy spaces $H_{L}(X)$ in [18].

3.1.1. The atomic Hardy spaces $H_{L, a t, M}(X)$. We now recall the notion of a $(1,2, M)$-atom associated to operators on spaces $(X, d, \mu)$. In what follows, denote by $\mathcal{D}(T)$ the domain of an operator $T$.

Definition 3.1 ([18]). A function $a(x) \in L^{2}(X)$ is called a $(1,2, M)$-atom associated to an operator $L$ if there exist a function $b \in \mathcal{D}\left(L^{M}\right)$ and a ball $B$ of $X$ such that

(i) $a=L^{M} b$;

(ii) $\operatorname{supp} L^{k} b \subset B, k=0,1, \ldots, M$;

(iii) $\left\|\left(r_{B}^{2} L\right)^{k} b\right\|_{L^{2}(X)} \leq r_{B}^{2 M} V(B)^{-1 / 2}, k=0,1, \ldots, M$. 
In the case $\mu(X)<\infty$ the constant function having value $[\mu(X)]^{-1}$ is also considered to be an atom.

Definition 3.2. Given $M>n / 4$, the atomic Hardy space $H_{L, a t, M}(X)$ is defined as follows. We shall say that $f=\sum \lambda_{j} a_{j}$ is an atomic $(1,2, M)$-representation if $\left\{\lambda_{j}\right\}_{j=0}^{\infty} \in l^{1}$, each $a_{j}$ is a $(1,2, M)$-atom, and the sum converges in $L^{2}(X)$. Set

$$
\boldsymbol{H}_{L, a t, M}(X)=\{f ; f \text { has an atomic }(1,2, M) \text {-representation }\},
$$

with the norm given by

$$
\|f\|_{\boldsymbol{H}_{L, a t, M}(X)}=\inf \left\{\sum_{j=0}^{\infty}\left|\lambda_{j}\right| ; f=\sum_{j=0}^{\infty} \lambda_{j} a_{j} \text { is an atomic (1,2,M)-representation }\right\} .
$$

The space $H_{L, a t, M}(X)$ is then defined as the completion of $\boldsymbol{H}_{L, a t, M}(X)$ with respect to the quasi-metric $d$ defined by $d(h, g)=\|h-g\|_{\boldsymbol{H}_{L, a t, M}(X)}$ for $h, g \in \boldsymbol{H}_{L, a t, M}(X)$.

A basic result concerning these spaces is the following proposition.

Proposition 3.3 ([18]). If an operator L satisfies conditions (H1) and (H2), then for all integers $M \in N$ with $M>n / 4$, the spaces $H_{L, a t, M}(X)$ coincide and their norms are equivalent.

We next recall the notion of a $(1,2, M, \varepsilon)$-molecule associated to an operator $L$.

Definition 3.4 ([18]). Let $\varepsilon>0$ and $M \in N$. We say that a function $\alpha \in L^{2}(X)$ is called a $(1,2, M, \varepsilon)$-molecule associated to $L$ if there exist a function $b \in \mathcal{D}\left(L^{M}\right)$ and a ball $B$ such that

(i) $\alpha=L^{M} b$;

(ii) $\left\|\left(r_{B}^{2} L\right)^{k} b\right\|_{L^{2}\left(S_{j}(B)\right)} \leq r_{B}^{2 M} 2^{-j \varepsilon} V\left(2^{j} B\right)^{-1 / 2}$, for all $k=0,1, \ldots, M$ and $j \in N$.

DEFinition 3.5. We shall say that $f=\sum \lambda_{j} a_{j}$ is an atomic $(1,2, M)$-representation if $\left\{\lambda_{j}\right\}_{j=0}^{\infty} \in l^{1}$, each $a_{j}$ is a $(1,2, M, \varepsilon)$-molecule, and the sum converges in $L^{2}(X)$. Set

$$
\boldsymbol{H}_{L, m o l, M}(X)=\{f ; f \text { has a molecular }(1,2, M, \varepsilon) \text {-representation }\}
$$

with the norm given by

$$
\|f\|_{\boldsymbol{H}_{L, m o l, M}(X)}=\inf \left\{\sum_{j=0}^{\infty}\left|\lambda_{j}\right| ; f=\sum_{j=0}^{\infty} \lambda_{j} a_{j} \text { is a molecular }(1,2, M, \varepsilon) \text {-representation }\right\} .
$$

The space $H_{L, m o l, M}(X)$ is then defined as the completion of $\boldsymbol{H}_{L, m o l, M}(X)$ with respect to the quasi-metric $d$ defined by $d(h, g)=\|h-g\|_{\boldsymbol{H}_{L, m o l, M}(X)}$ for $h, g \in \boldsymbol{H}_{L, m o l, M}(X)$.

3.2. A characterization of Hardy spaces in terms of square functions. Set $H^{2}(X)=\overline{\mathcal{R}(L)}$, where $\mathcal{R}(L):=\left\{L u ; u \in L^{2}(X)\right\}$. The space $H_{L, S_{L}}(X)$ is defined as the completion of

$$
\left\{f \in H^{2}(X) ;\left\|S_{L} f\right\|_{L^{1}(X)}<\infty\right\}
$$


with respect to the norms given by

$$
\|f\|_{H_{L, S_{L}}^{1}(X)}=\left\|S_{h} f\right\|_{L^{1}(X)}
$$

where

$$
S_{L} f(x):=\left(\int_{0}^{\infty} \int_{d(x, y)<t}\left|t^{2} L e^{-t^{2} L} f(y)\right|^{2} \frac{d \mu(y)}{V(x, t)} \frac{d t}{t}\right)^{1 / 2} .
$$

Then the Hardy spaces $H_{L, S_{L}}, H_{L, a t, M}(X)$ and $H_{L, m o l, M}(X)$ are equivalent, if the parameter $M>n / 4$. In fact, we have the following result.

Proposition 3.6 ([18]). Suppose $M>n / 4$. Then $H_{L, S_{L}}(X)=H_{L, a t, M}(X)=$ $H_{L, m o l, M}(X)$ and their norms are equivalent.

Consequently, as in the next definition, when $M>n / 4$, one may write $H_{L, a t}$ and $H_{L, m o l}$ in place of $H_{L, a t, M}$ and $H_{L, m o l, M}$, respectively. Precisely, we have the following definition.

Definition 3.7. The Hardy space $H_{L}(X)$ is defined by

$$
H_{L}(X):=H_{L, S_{L}}(X) .
$$

Note that for $M>n / 4, H_{L}(X)$ is identical with $H_{L, a t, M}(X):=H_{L, m o l, M}(X)$.

3.3. The space $\mathrm{BMO}_{L}(X)$. Let $\phi=L^{M} v$ be a function in $L^{2}(X)$, where $v \in D\left(L^{M}\right)$. Following $[17,18]$ for $\varepsilon>0, M \in N$ and fixed $x_{0} \in X$ we introduce the norm

$$
\|\phi\|_{\mathcal{M}_{0}^{M, \varepsilon}(L)}=\sup _{j \in \boldsymbol{Z}_{+}}\left\{2^{j \varepsilon} V\left(x_{0}, 2^{j}\right)^{1 / 2} \sum_{k=0}^{M}\left\|L^{k} v\right\|_{L^{2}\left(S_{j}\left(B\left(x_{0}, 1\right)\right)\right)}\right\}
$$

and the set

$$
\mathcal{M}_{0}^{M, \varepsilon}(L)=\left\{\phi=L^{M} v \in L^{2}(X) ;\|\phi\|_{\mathcal{M}_{0}^{M, \varepsilon}(L)}<\infty\right\} .
$$

Let $\left(\mathcal{M}_{0}^{M, \varepsilon}(L)\right)^{*}$ be the dual of $\mathcal{M}_{0}^{M, \varepsilon}(L)$, and denote either $\left(I+t^{2} L\right)^{-1}$ or $e^{-t^{2} L}$ by $A_{t}$. Let $f \in\left(\mathcal{M}_{0}^{M, \varepsilon}(L)\right)^{*}$. Then $\left(I-A_{t}\right)^{M} f$ belongs to $L_{\text {loc }}^{2}(X)$ in the sense of distribution (see $[17,18])$.

For any $M \in N$ define

$$
\mathcal{E}_{M}=\bigcap_{\varepsilon>0}\left(\mathcal{M}_{0}^{M, \varepsilon}(L)\right)^{*} .
$$

Definition 3.8 ([18]). Let $L$ satisfy assumptions (H1) and (H2) and $M>n / 4$. A functional $f \in \mathcal{E}_{M}$ is said to be in $\mathrm{BMO}_{L, M}(X)$ if

$$
\|f\|_{\mathrm{BMO}_{L, M}(X)}=\sup _{B \subset X}\left[\frac{1}{V(B)} \int_{B}\left|\left(I-e^{-r_{B}^{2} L}\right)^{M} f(x)\right|^{2} d \mu(x)\right]^{1 / 2}<\infty,
$$

where the supremum is taken over all balls $B$ of $X$.

We have the following characterizations of the spaces $\mathrm{BMO}_{L, M}(X)$. 
Proposition 3.9 ([18]). Let L satisfy assumptions (H1) and (H2) and $M>n / 4$. Then $f \in \mathrm{BMO}_{L, M}(X)$ if and only if $f \in \mathcal{E}_{M}$ and

$$
\|f\|_{\mathrm{BMO}_{L, M}(X)}=\sup _{B \subset X}\left[\frac{1}{V(B)} \int_{B}\left|\left(I-\left(I+r_{B}^{2} L\right)^{-1}\right)^{M} f(x)\right|^{2} d \mu(x)\right]^{1 / 2}<\infty .
$$

Moreover,

$$
\|f\|_{\mathrm{BMO}_{L, M}(X)} \approx \sup _{B \subset X}\left[\frac{1}{V(B)} \int_{B}\left|\left(I-\left(I+r_{B}^{2} L\right)^{-1}\right)^{M} f(x)\right|^{2} d \mu(x)\right]^{1 / 2} .
$$

Since the spaces $\mathrm{BMO}_{L, M}(X)$ coincide for all $M>n / 4$ (see [18]), in what follows, we denote $\mathrm{BMO}_{L, M}(X)$ simply by $\mathrm{BMO}_{L}(X)$.

Proposition 3.10. Let L satisfy assumptions $(\mathrm{H} 1),(\mathrm{H} 2)$ and $M>n / 4$. Then there exists a positive constant $C$ such that for all $f \in \mathrm{BMO}_{L, M}(X)$,

$$
\sup _{B \subset X}\left[\frac{1}{V(B)} \int_{\widehat{B}}\left|\left(t^{2} L\right)^{M} e^{-t^{2} L} f(x)\right|^{2} \frac{d \mu(x) d t}{t}\right]^{1 / 2} \leq C\|f\|_{\mathrm{BMO}_{L, M}(X)} .
$$

Conversely, for $\tilde{M}>M+n / 4$, if $f \in \mathcal{E}_{\widetilde{M}}$ and

$$
\sup _{B \subset X}\left[\frac{1}{V(B)} \int_{\widehat{B}}\left|\left(t^{2} L\right)^{\widetilde{M}} e^{-t^{2} L} f(x)\right|^{2} \frac{d \mu(x) d t}{t}\right]^{1 / 2}<\infty,
$$

then $f \in \mathrm{BMO}_{L}(X)$.

For the proof of (5) and (6) we refer the reader to [19, Proposition 4.5] and [17, Lemma 8.3], respectively.

3.4. The space $\mathrm{VMO}_{L}(X)$.

Definition 3.11. Let $L$ satisfy (H1) and (H2) and $M>n / 4$. A function $f \in$ $\mathrm{BMO}_{L}(X)$ is said to be in $\mathrm{VMO}_{L, M}(X)$ if $\gamma_{1}(f)=\gamma_{2}(f)=\gamma_{3}(f)=0$, where

$$
\begin{aligned}
& \gamma_{1}(f)=\lim _{a \rightarrow 0} \sup _{B: r_{B}<a}\left[\frac{1}{V(B)} \int_{B}\left|\left(I-e^{-r_{B}^{2} L}\right)^{M} f(x)\right|^{2} d \mu(x)\right]^{1 / 2}, \\
& \gamma_{2}(f)=\lim _{b \rightarrow \infty} \sup _{B: r_{B}>b}\left[\frac{1}{V(B)} \int_{B}\left|\left(I-e^{-r_{B}^{2} L}\right)^{M} f(x)\right|^{2} d \mu(x)\right]^{1 / 2},
\end{aligned}
$$

and

$$
\gamma_{3}(f)=\lim _{c \rightarrow \infty} \sup _{B: B \subset B\left(x_{0}, c\right)^{c}}\left[\frac{1}{V(B)} \int_{B}\left|\left(I-e^{-r_{B}^{2} L}\right)^{M} f(x)\right|^{2} d \mu(x)\right]^{1 / 2} .
$$

For $f \in \mathrm{VMO}_{L, M}(X)$, define $\|f\|_{\mathrm{VMO}_{L, M}(X)}:=\|f\|_{\mathrm{BMO}_{L}(X)}$.

Proposition 3.12. Assume that $M>n / 4$ and $f \in \mathrm{BMO}_{L}(X)$. Then, $f \in$ $\operatorname{VMO}_{L, M}(X)$ iff $\tilde{\gamma_{1}}(f)=\tilde{\gamma_{2}}(f)=\tilde{\gamma_{3}}(f)=0$, where

$$
\tilde{\gamma_{1}}(f)=\lim _{a \rightarrow 0} \sup _{B: r_{B}<a}\left[\frac{1}{V(B)} \int_{B}\left|\left(I-\left(I+r_{B}^{2} L\right)^{-1}\right)^{M} f(x)\right|^{2} d \mu(x)\right]^{1 / 2},
$$


(8) $\quad \tilde{\gamma_{2}}(f)=\lim _{b \rightarrow \infty} \sup _{B: r_{B}>b}\left[\frac{1}{V(B)} \int_{B}\left|\left(I-\left(I+r_{B}^{2} L\right)^{-1}\right)^{M} f(x)\right|^{2} d \mu(x)\right]^{1 / 2}$,

and

(9) $\quad \tilde{\gamma}_{3}(f)=\lim _{c \rightarrow \infty} \sup _{B: B \subset B\left(x_{0}, c\right)^{c}}\left[\frac{1}{V(B)} \int_{B}\left|\left(I-\left(I+r_{B}^{2} L\right)^{-1}\right)^{M} f(x)\right|^{2} d \mu(x)\right]^{1 / 2}$.

Proof. For the sake of simplicity, we denote the set of all functions in $\operatorname{BMO}_{L}(X)$ satisfying (7), (8) and (9) by $\mathrm{VMO}^{\mathrm{res}}{ }_{L, M}(X)$.

STEP 1. $\mathrm{VMO}_{L, M}^{\text {res }}(X) \subset \mathrm{VMO}_{L, M}(X)$.

For $f \in \mathrm{VMO}_{L, M}^{\text {res }}(X)$, we break

$$
f=\left(I-\left(I+r_{B}^{2} L\right)^{-1}\right)^{M} f+\left(I-\left(I-\left(I+r_{B}^{2} L\right)^{-1}\right)^{M}\right) f .
$$

Then, for any ball $B \subset X$, we have

$$
\begin{aligned}
I_{1}:= & \left(\frac{1}{V(B)} \int_{B}\left|\left(I-e^{-r_{B}^{2} L}\right)^{M}\left(I-\left(I+r_{B}^{2} L\right)^{-1}\right)^{M} f(x)\right|^{2} d \mu(x)\right)^{1 / 2} \\
\leq & C \sum_{k=0}^{M} \sum_{j=0}^{\infty}\left(\frac{1}{V(B)} \int_{B}\left|e^{-k r_{B}^{2} L}\left[\chi_{S_{j}(B)}\left(I-\left(I+r_{B}^{2} L\right)^{-1}\right)^{M} f\right](x)\right|^{2} d \mu(x)\right)^{1 / 2} \\
\leq & \left(\frac{1}{V(B)} \int_{B}\left|\left(I-\left(I+r_{B}^{2} L\right)^{-1}\right)^{M} f(x)\right|^{2} d \mu(x)\right)^{1 / 2} \\
& +C \sum_{j=2}^{\infty} e^{-c 2^{j}}\left(\frac{1}{V(B)} \int_{S_{j}(B)}\left|\left(I-\left(I+r_{B}^{2} L\right)^{-1}\right)^{M} f(x)\right|^{2} d \mu(x)\right)^{1 / 2} .
\end{aligned}
$$

At this stage, by Lemma 2.1 and Remark 2.2, $I_{1}$ is dominated by

$$
C \sum_{j=0}^{\infty} e^{-c 2^{j}} 2^{(n+N) j / 2} \delta_{j}(f, B)
$$

where

$$
\delta_{j}(f, B):=\sup _{B^{\prime}: B^{\prime} \subset 2^{j+1} B, r_{B^{\prime}}=r_{B}}\left(\frac{1}{V\left(B^{\prime}\right)} \int_{B^{\prime}}\left|\left(I-\left(I+r_{B}^{2} L\right)^{-1}\right)^{M} f(x)\right|^{2} d \mu(x)\right)^{1 / 2} .
$$

To estimate the term

$$
I_{2}:=\left(\frac{1}{V(B)} \int_{B}\left|\left(I-e^{-r_{B}^{2} L}\right)^{M}\left(I-\left(I-\left(I+r_{B}^{2} L\right)^{-1}\right)^{M}\right) f(x)\right|^{2} d \mu(x)\right)^{1 / 2},
$$

we write

$$
I-\left(I-\left(I+r_{B}^{2} L\right)^{-1}\right)^{M}=\sum_{k=1}^{M} \frac{M !}{(M-k) ! k !}\left(r_{B}^{2} L\right)^{-k}\left(I-\left(I+r_{B}^{2} L\right)^{-1}\right)^{M} .
$$


Therefore,

$$
\begin{aligned}
I_{2} \leq C \sum_{j=1}^{M}( & \frac{1}{V(B)} \int_{B} \mid\left(I-e^{-r_{B}^{2} L}\right)^{M-k}\left(-\int_{0}^{r_{B}} \frac{\tau}{r_{B}^{2}} e^{-\tau^{2} L} d \tau\right)^{j} \\
& \left.\times\left.\left(r_{B}^{2} L\right)^{-k}\left(I-\left(I+r_{B}^{2} L\right)^{-1}\right)^{M} f(x)\right|^{2} d \mu(x)\right)^{1 / 2} .
\end{aligned}
$$

By changing the order of integration above, using the annular decomposition and DaviesGaffney estimates and repeating the argument above, we also obtain

$$
I_{2} \leq C \sum_{j=0}^{\infty} e^{-c 2^{j}} 2^{(n+N) j / 2} \delta_{j}(f, B) .
$$

Therefore,

$$
\begin{aligned}
& \left(\frac{1}{V(B)} \int_{B}\left|\left(I-e^{-r_{B}^{2} L}\right)^{M} f(x)\right|^{2} d \mu(x)\right)^{1 / 2} \leq C \sum_{j=0}^{\infty} e^{-c 2^{j}} 2^{(n+N) j / 2} \delta_{j}(f, B) \\
& \quad \leq C \sum_{j=0}^{k_{0}} e^{-c 2^{j}} 2^{(n+N) j / 2} \delta_{j}(f, B)+C \sum_{k_{0}}^{\infty} e^{-c 2^{j}} 2^{(n+N) j / 2}\|f\|_{\mathrm{BMO}_{L}} \\
& \quad \leq C \sum_{j=0}^{k_{0}} e^{-c 2^{j}} 2^{(n+N) j / 2} \delta_{j}(f, B)+C e^{-c 2_{0}^{k}} 2^{(n+N) k_{0} / 2}\|f\|_{\mathrm{BMO}_{L}} .
\end{aligned}
$$

So, when $k_{0}$ is sufficiently large, $\gamma_{1}(f)=\gamma_{2}(f)=\gamma_{3}(f)=0$. This implies $f \in$ $\mathrm{VMO}_{L, M}(X)$.

STEP 2. $\mathrm{VMO}_{L, M}(X) \subset \mathrm{VMO}^{\text {res }}{ }_{L, M}(X)$.

The proof of this step is just a simple combination one in Step 1 and one of [17, Lemma 8.1] and hence we omit detail here.

3.5. Characterization of $\mathrm{VMO}_{L}(X)$ via tent spaces. For any $x \in X$ and $\alpha>0$, the cone of aperture $\alpha$ and vertex $x$ is the set

$$
\Gamma^{\alpha}(x)=\{(y, t) \in X \times(0, \infty) ; d(y, x)<\alpha t\} .
$$

For any closed subset $F \subset X$, define a saw-tooth region $R^{\alpha}(F)=\bigcup_{x \in F} \Gamma^{\alpha}(x)$. For simplicity, we will often write $R(F)$ instead of $R^{1}(F)$. If $O$ is an open subset of $X$, and we denote by $E^{c}$ the complement of a set $E$, then the tent over $O$, denoted by $\widehat{O}$, is defined as

$$
\widehat{O}=\left[R\left(O^{c}\right)\right]^{c}=\left\{(x, t) \in X \times(0, \infty) ; d\left(x, O^{c}\right)<\alpha t\right\} .
$$

Lemma 3.13 ([18]). For a measurable function $F$ defined on $X \times(0, \infty)$, define

$$
\mathcal{A}^{\alpha}(F)(x)=\left(\int_{\Gamma^{\alpha}(x)}|F(y, t)|^{2} \frac{d \mu(y)}{V(x, t)} \frac{d t}{t}\right)^{1 / 2}, \alpha>0,
$$


and set $\mathcal{A}(F)(x)=\mathcal{A}^{1}(F)(x)$. Then there exists a constant $C>0$ depending only on $n$ and $N$ such that

$$
\left\|\mathcal{A}^{\alpha}(F)\right\|_{L^{1}} \leq C\|\mathcal{A}(F)\|_{L^{1}} .
$$

When $X=\boldsymbol{R}^{n}$ Coifman, Meyer and Stein [9] introduced the tent space $T_{2}^{p}\left(\boldsymbol{R}_{+}^{n+1}\right)$ for $p \in(0, \infty)$. The tent space $T_{2}^{p}(X)$ on homogeneous type spaces was studied by Russ [22]. A function $F$ is said to belong to the spaces $T_{2}^{p}(X)$ with $p \in(0, \infty)$ if $\|F\|_{T_{2}^{p}(X)}=$ $\|\mathcal{A}(F)\|_{L^{p}}<\infty$. We define

$$
\mathcal{C}(F)(x)=\sup _{B \ni x}\left(\frac{1}{V(B)} \int_{\widehat{B}}|F(y, t)|^{2} d \mu(y) \frac{d t}{t}\right)^{1 / 2},
$$

where the supremum is taken over all balls $B$ containing $x$ and we say that $F \in T_{2}^{\infty}(X)$ if $\|F\|_{T_{2}^{\infty}(X)}=\|\mathcal{C}(F)\|_{L^{\infty}}<\infty$. Next, a function $a(x, t)$ is called a $T_{2}^{1}(X)$-atom if

(i) the function $a(x, t)$ is supported in $\widehat{B}$ (for some ball $B$ );

(ii) $\int_{\widehat{B}}|a(x, t)|^{2} d \mu(x) \frac{d t}{t} \leq \frac{1}{V(B)}$.

We now state some basic facts for tent spaces, which are shown in [9] for $\boldsymbol{R}^{n}$ and in [4] for the spaces of homogenous type.

Proposition 3.14. (i) There exists $C>0$ such that for all $F \in T_{2}^{1}(X)$ and all $G \in T_{2}^{\infty}(X)$,

$$
\int_{X \times(0, \infty)}|F(x, t) G(x, t)| d \mu(x) \frac{d t}{t} \leq C \int_{X} \mathcal{A}(F)(x) \mathcal{C}(G)(x) d \mu(x) .
$$

(ii) The paring $\langle F, G\rangle \mapsto \int_{X \times(0, \infty)} F(x, t) G(x, t) d \mu(x) \frac{d t}{t}$ realizes $T_{2}^{\infty}(X)$ as equivalent with the dual of $T_{2}^{1}(X)$.

In what follows, let $T_{2, b}^{p}$ be the set of all $f \in T_{2}^{p}$ with bounded support in $X \times(0, \infty)$. We denote by $T_{2,0}^{\infty}$ the linear subspace of $T_{2}^{\infty}$ consisting of those functions satisfying the condition

$$
v_{1}(f)=\lim _{a \rightarrow 0}\left[\sup _{B: r_{B} \leq a} \frac{1}{V(B)} \int_{\widehat{B}}|f(y, t)|^{2} d \mu(y) \frac{d t}{t}\right]=0,
$$

and we endow $T_{2,0}^{\infty}$ with norm of $T_{2}^{\infty}$. Then, we denote by $T_{2, V}^{\infty}$ the closure of the set $T_{2, b}^{\infty}$ in $T_{2,0}^{\infty}$ with respect to the norm of $T_{2}^{\infty}$.

Let $\mathcal{T}$ be the set of all functions satisfying the three following conditions:

(i) $f \in T_{2,0}^{\infty}$;

(ii) $v_{2}(f)=\lim _{b \rightarrow \infty}\left[\sup _{B: r_{B} \geq b} \frac{1}{V(B)} \int_{\widehat{B}}|f(y, t)|^{2} d \mu(y) \frac{d t}{t}\right]=0$,

(iii) $v_{3}(f)=\lim _{c \rightarrow \infty}\left[\sup _{B: B \subset B\left(x_{0}, c\right)^{c}} \frac{1}{V(B)} \int_{\widehat{B}}|f(y, t)|^{2} d \mu(y) \frac{d t}{t}\right]=0$.

We can verify that $\mathcal{T}$ is a closed linear subspace of $T_{2}^{\infty}$. Note that conditions (ii) and (iii) are not consequences of (i), see [12]. The following lemma show that the coincidence of $\mathcal{T}$ and $T_{2, V}^{\infty}$. Let us remind that the similar result was obtained in [12], but in $\boldsymbol{R}^{n}$ setting. 
LEMMA 3.15. The following statements hold:

(i) $\left(T_{2, V}^{\infty}\right)^{*}=T_{2}^{1}$;

(ii) $f \in T_{2, V}^{\infty}$ iff $f \in \mathcal{T}$.

PROOF. The proof of (i) is analogous to [24, Theorem 1.7] and hence we omit the detail here.

(ii) It is easy to see that $T_{2, b}^{\infty} \subset \mathcal{T}$. This together with $\mathcal{T}$ is closed on $T_{2}^{\infty}$ gives $T_{2, V}^{\infty} \subset \mathcal{T}$. Conversely, for $f \in \mathcal{T}$, by definition, for any $\varepsilon$ there exist $a_{0}, b_{0}, c_{0}$ such that

$$
\begin{aligned}
& \sup _{B: r_{B} \leq a_{0}} V(B)^{-1} \int_{\widehat{B}}|f(y, t)|^{2} d \mu(y) \frac{d t}{t} \leq \varepsilon, \\
& \sup _{B: r_{B} \geq b_{0}} V(B)^{-1} \int_{\widehat{B}}|f(y, t)|^{2} d \mu(y) \frac{d t}{t} \leq \varepsilon,
\end{aligned}
$$

and

$$
\sup _{B: B \subset B\left(x_{0}, c_{0}\right)^{c}} V(B)^{-1} \int_{\widehat{B}}|f(y, t)|^{2} d \mu(y) \frac{d t}{t} \leq \varepsilon .
$$

By standard argument, set $K_{0}=\max \left\{a_{0}^{-1}, b_{0}, c_{0}\right\}$ and define

$$
g(y, t)=f(y, t) \chi_{B\left(0,2 K_{0}\right) \times\left(\left(2 K_{0}\right)^{-1}, 2 K_{0}\right)}(y, t) .
$$

Then, $g \in T_{2, b}^{2}$. To complete the proof, we need to show that $\|f-g\|_{T_{2}^{\infty}}^{2} \leq c \varepsilon$. We consider the following three cases:

CASE 1: $\quad r_{B}<a_{0}$ or $r_{B}>b_{0}$. We have,

$$
\|f-g\|_{T_{2}^{\infty}}^{2} \leq 2 \leq 2 V(B)^{-1} \int_{\widehat{B}}|f(y, t)|^{2} d \mu(y) \frac{d t}{t} \leq 2 \varepsilon .
$$

CASE 2: $\quad a_{0} \leq r_{B}<b_{0}$ and $B \subset B\left(x_{0}, c_{0}\right)^{c}$. We have,

$$
\|f-g\|_{T_{2}^{\infty}}^{2} \leq 2 V(B) \int_{\widehat{B}}|f(y, t)|^{2} d \mu(y) \frac{d t}{t} \leq 2 \varepsilon .
$$

CASE 3: $a_{0} \leq r_{B}<b_{0}$ and $B \cap B\left(x_{0}, c_{0}\right)^{c} \neq \emptyset$. By a simple calculation, one has

$$
\begin{aligned}
\int_{\widehat{B}}|f(y, t)-g(y, t)|^{2} d \mu(y) \frac{d t}{t} & \leq \int_{0}^{\left(2 K_{0}\right)^{-1}} \int_{B}|f(y, t)|^{2} d \mu(y) \frac{d t}{t} \\
& \leq \int_{0}^{\left(2 K_{0}\right)^{-1}} \int_{B\left(x_{B}, 2^{k} a_{0}\right)}|f(y, t)|^{2} d \mu(y) \frac{d t}{t}
\end{aligned}
$$

where $k$ is the smallest integer such that $2^{k} a_{0} \geq r_{B}$.

At this stage, applying Lemma 2.1, we can pick an almost disjoint family of balls with the same radius $a_{0},\left\{B_{k_{1}}, \ldots, B_{k_{N_{k}}}\right\}$ such that $B\left(x_{B}, 2^{k} a_{0}\right) \subset \cup_{j=1}^{N_{k}} B_{k_{j}}, N_{k} \leq C 2^{-k n}$ and $\sum_{j=1}^{N_{k}} \chi_{B_{k_{j}}} \leq C_{0}\left(C_{0}\right.$ is independent of $k$ and $\left.a_{0}\right)$. Therefore,

$$
\int_{\widehat{B}}|f(y, t)-g(y, t)|^{2} d \mu(y) \frac{d t}{t} \leq \int_{0}^{\left(2 K_{0}\right)^{-1}} \int_{\cup_{j=1}^{N_{k}} B_{k_{j}}}|f(y, t)|^{2} d \mu(y) \frac{d t}{t}
$$




$$
\begin{aligned}
& \leq \sum_{j=1}^{N_{k}} \int_{0}^{\left(2 K_{0}\right)^{-1}} \int_{B_{k_{j}}}|f(y, t)|^{2} d \mu(y) \frac{d t}{t} \\
& \leq \sum_{j=1}^{N_{k}} \int_{\widehat{B_{k_{j}}}}|f(y, t)|^{2} d \mu(y) \frac{d t}{t} \\
& \leq c \varepsilon \sum_{j=1}^{N_{k}} \mu\left(B_{k_{j}}\right) \\
& \leq c \varepsilon \mu(2 B) \\
& \leq c \varepsilon \mu(B) .
\end{aligned}
$$

This completes our proof.

Proposition 3.16. Let L satisfy (H1) and (H2). Assume that $M>n / 4$ and $\tilde{M}>$ $M+n / 4$. Then the following statements are equivalent

(a) $f \in \mathrm{VMO}_{L, M}(X)$;

(b) $f \in \mathcal{E}_{\tilde{M}}$ and $\left(t^{2} L\right)^{\tilde{M}} e^{-t^{2} L} f \in T_{2, V}^{\infty}$.

Therefore, $\left\|\left(t^{2} L\right)^{\widetilde{M}} e^{-t^{2} L} f\right\|_{T_{2, V}^{\infty}} \approx\|f\|_{\mathrm{BMO}_{L}(X)}$.

Note that from this proposition, all spaces $\operatorname{VMO}_{L, M}(X)$ are coincide provided $M>n / 4$. So, for the rest of the paper, we write simply $\operatorname{VMO}_{L}(X)$ instead of $\operatorname{VMO}_{L, M}(X)$.

Proof. (a) $\Rightarrow$ (b): Since $f \in \mathrm{VMO}_{L, M}(X), f \in \mathrm{BMO}_{L, M}(X)$. By Proposition 3.10, $\left(t^{2} L\right)^{\tilde{M}} e^{-t^{2} L} f \in T_{2}^{\infty}$. To show $\left(t^{2} L\right)^{\widetilde{M}} e^{-t^{2} L} f \in T_{2, V}^{\infty}$, by similar argument in the proof of Proposition 3.12, it is sufficient to claim that, for any ball $B \subset X$, there holds

$$
\left(\frac{1}{V(B)} \int_{\widehat{B}}\left|\left(t^{2} L\right)^{\widetilde{M}} e^{-t^{2} L} f\right|^{2} d \mu(y) \frac{d t}{t}\right)^{1 / 2} \leq C \delta_{0}(f, 4 B)+C \sum_{j=0}^{\infty} 2^{-j / 2} \delta_{j}(f, B) .
$$

To do this, we put $f_{1}=\left(I-\left(I+r_{B}^{2} L\right)^{-1}\right)^{\widetilde{M}} f$ and $f_{2}=f-f_{1}$. We then have

$$
\begin{aligned}
\left(\frac{1}{V(B)}\right. & \left.\int_{\widehat{B}}\left|\left(t^{2} L\right)^{\widetilde{M}} e^{-t^{2} L} f_{1}\right|^{2} d \mu(y) \frac{d t}{t}\right)^{1 / 2} \\
\leq & \sum_{j=0}^{\infty}\left(\frac{1}{V(B)} \int_{\widehat{B}}\left|\left(t^{2} L\right)^{\widetilde{M}} e^{-t^{2} L} f_{1} \chi_{S_{j}(B)}\right|^{2} d \mu(y) \frac{d t}{t}\right)^{1 / 2} \\
\leq & C\left(\frac{1}{V(4 B)} \int_{4 B}\left|f_{1}\right|^{2} d \mu(x)\right)^{1 / 2} \\
& +\sum_{j=2}^{\infty} \frac{1}{V(B)^{1 / 2}}\left(\int_{S_{j}(B)}\left|f_{1}\right|^{2} d \mu(x)\right)^{1 / 2}\left(\int_{0}^{r_{B}} \exp \left\{-\frac{\left(2^{j} r_{B}\right)^{2}}{c t^{2}}\right\} \frac{d t}{t}\right)^{1 / 2}
\end{aligned}
$$




$$
\begin{aligned}
\leq & C \delta_{0}(f, 4 B)+\sum_{j=2}^{\infty} \frac{1}{V(B)^{1 / 2}}\left(\int_{S_{j}(B)}\left|f_{1}\right|^{2} d \mu(x)\right)^{1 / 2} \\
& \times\left(\int_{0}^{r_{B}}\left[\frac{c t^{2}}{\left(2^{j} r_{B}\right)^{2}}\right]^{(n+N+1) / 2} \frac{d t}{t}\right)^{1 / 2} \\
\leq & C \delta_{0}(f, 4 B)+\sum_{j=2}^{\infty}\left(\frac{2^{-j(n+N+1)}}{V(B)} \int_{S_{j}(B)}\left|f_{1}\right|^{2} d \mu(x)\right)^{1 / 2} .
\end{aligned}
$$

Applying Lemma 2.1 and Remark 2.2, we can pick the almost disjoint family of balls $\left\{B\left(x_{k_{1}}, r_{B}\right), \ldots, B\left(x_{k_{N_{k}}}, r_{B}\right)\right\}$ which covers $2^{j} B$ satisfying $N_{k} \leq C 2^{j n}$ and $V(B)^{-1} \leq$ $2^{j N} V\left(x_{k_{j}}, r_{B}\right)$. Therefore, we obtain

$$
\begin{aligned}
& \left(\frac{1}{V(B)} \int_{\widehat{B}}\left|\left(t^{2} L\right)^{\widetilde{M}} e^{-t^{2} L} f_{1}\right|^{2} d \mu(y) \frac{d t}{t}\right)^{1 / 2} \\
& \leq C \delta_{0}(f, 4 B)+\sum_{j=2}^{\infty}\left(\sum_{j=1}^{N_{k}} \frac{2^{-j(n+1)}}{V\left(x_{k_{j}}, r_{B}\right)} \int_{B_{x_{k_{j}}, r_{B}}}\left|f_{1}\right|^{2} d \mu(x)\right)^{1 / 2} \\
& \leq C \delta_{0}(f, 4 B)+C \sum_{j=0}^{\infty} 2^{-j / 2} \delta_{j}(f, B) .
\end{aligned}
$$

By similar argument above, by using the decomposition as in Proposition 3.12

$$
I-\left(I-\left(I+r_{B}^{2} L\right)^{-1}\right)^{M}=\sum_{k=1}^{M} \frac{M !}{(M-k) ! k !}\left(r_{B}^{2} L\right)^{-k}\left(I-\left(I+r_{B}^{2} L\right)^{-1}\right)^{M} .
$$

We also get that

$$
\left(\frac{1}{V(B)} \int_{\widehat{B}}\left|\left(t^{2} L\right)^{\widetilde{M}} e^{-t^{2} L} f_{2}\right|^{2} d \mu(y) \frac{d t}{t}\right)^{1 / 2} \leq C \delta_{0}(f, 4 B)+C \sum_{j=0}^{\infty} 2^{-j / 2} \delta_{j}(f, B) .
$$

This follows therefore (b).

(b) $\Rightarrow$ (a) : From $f \in \mathcal{E}_{\tilde{M}}$ an $\left(t^{2} L\right)^{\tilde{M}} e^{-t^{2} L} f \in T_{2, V}^{\infty}$ together with Proposition 3.10, $f \in$ $\mathrm{BMO}_{L(X)}$. Let $g \in L^{2}(B)$ with norm 1. By using the argument in [17, p. 43], $\left(I-e^{-r_{B}^{2} L}\right)^{M} g$ is a multiple $(1,2, M, \varepsilon)$-molecule. Therefore, one has

$$
\begin{aligned}
\left(\int_{B}\right. & \left.\left|\left(I-e^{-r_{B}^{2} L}\right)^{M} f\right|^{2} d \mu(x)\right)^{1 / 2} \\
= & \sup _{\|g\|_{L^{2}(B)}=1} \int_{B} f(x)\left(I-e^{-r_{B}^{2} L}\right)^{M} g(x) d \mu(x) \\
= & \sup _{\|g\|_{L^{2}(B)}} \iint_{X \times(0, \infty)}\left(t^{2} L\right)^{\widetilde{M}} e^{-t^{2} L} f(x) t^{2} L e^{-t^{2} L}\left(I-e^{-r_{B}^{2} L}\right)^{M} g(x) d \mu(x) \frac{d t}{t}
\end{aligned}
$$




$$
\begin{aligned}
\leq & \sum_{j=0}^{\infty}\left(\int_{V_{j}(B)}\left|\left(t^{2} L\right)^{\tilde{M}} e^{-t^{2} L} f(x)\right|^{2} d \mu(x) \frac{d t}{t}\right)^{1 / 2} \\
& \times\left(\sup _{\|g\|_{L^{2}(B)}=1}\left(\int_{V_{j}(B)}\left|t^{2} L e^{-t^{2} L}\left(I-e^{-r_{B}^{2} L}\right)^{M} g(x)\right|^{2} d \mu(x) \frac{d t}{t}\right)^{1 / 2}\right) \\
\leq & \sum_{j=0}^{\infty} \sigma_{j}(f, B) \boldsymbol{A}_{j}
\end{aligned}
$$

where $V_{0}(B)=\widehat{B}$ and $V_{j}(B)=\widehat{2^{j} B} \backslash \widehat{2^{j-1} B}$. Note that in the first equality we apply Corollary 4.2 in [19]. We would like to point out that it was prove in [19, Corollary 4.2] that the first equality holds for all $(1,2, M)$ atoms. However, by a minor modification, the statement is still true with $(1,2, M, \varepsilon)$ molecules.

For $j=0,1,2$, it is easy to check that

$$
\boldsymbol{A}_{j} \leq \sup _{\|g\|_{L^{2}(B)}=1}\left\|\left(I-e^{-r_{B}^{2} L}\right)^{M} g(x)\right\|_{L^{2}} \leq C .
$$

For $j \geq 3$, we have, by Proposition 2.4,

$$
\begin{aligned}
& \left(\int_{V_{j}(B)}\left|t^{2} L e^{-t^{2} L}\left(I-e^{-r_{B}^{2} L}\right)^{M} g(x)\right|^{2} d \mu(x) \frac{d t}{t}\right)^{1 / 2} \\
& \quad=\left(\int_{V_{j}(B)}\left|t^{2} L e^{-t^{2} L}\left(\int_{0}^{r_{B}^{2}} \cdots \int_{0}^{r_{B}^{2}} L^{M} e^{-\left(\tau_{1}+\cdots+\tau_{M}\right) L} d \tau_{1} \cdots d \tau_{M}\right) g(x)\right|^{2} d \mu(x) \frac{d t}{t}\right)^{1 / 2} \\
& \quad \leq \int_{0}^{r_{B}^{2}} \cdots \int_{0}^{r_{B}^{2}}\left(\int_{V_{j}(B)}\left|t^{2} L^{M+1} e^{-\left(t^{2}+\tau_{1}+\cdots+\tau_{M}\right) L} g(x)\right|^{2} d \mu(x) \frac{d t}{t}\right)^{1 / 2} d \tau_{1} \cdots d \tau_{M} \\
& \quad \leq \int_{0}^{r_{B}^{2}} \cdots \int_{0}^{r_{B}^{2}}\left(\int_{2^{j-1} r_{B}}^{2^{j} r_{B}}\left|\frac{t^{4}\|g\|_{L^{2}(B)}^{2}}{\left(t^{2}+\tau_{1}+\cdots+\tau_{M}\right)^{2(M+1)}}\right|^{2} d \mu(x) \frac{d t}{t}\right)^{1 / 2} d \tau_{1} \cdots d \tau_{M} \\
& \leq 2^{-2 j M} .
\end{aligned}
$$

Therefore, $\boldsymbol{A}_{j} \leq 2^{-2 j M}$. Combining above estimates, we have

$$
\begin{aligned}
& \left(\frac{1}{V(B)} \int_{B}\left|\left(I-e^{-r_{B}^{2} L}\right)^{M} f\right|^{2} d \mu(x)\right)^{1 / 2} \\
& \quad \leq \sum_{j=0}^{\infty} 2^{-2 j M}\left[\frac{V\left(2^{j} B\right)}{V(B)}\right]^{1 / 2}\left[\frac{1}{V\left(2^{j} B\right)^{1 / 2}} \sigma_{j}(f, B)\right] \\
& \leq \sum_{j=0}^{\infty} 2^{-2 j(M-n / 4)}\left[\frac{1}{V\left(2^{j} B\right)^{1 / 2}} \sigma_{j}(f, B)\right]
\end{aligned}
$$

We then follow the argument as in the proof of the implication (a) $\Rightarrow$ (b) to show that $f \in$ $\mathrm{VMO}_{L, M}(X)$. 
4. Duality between $H_{L}(X)$ and $\operatorname{VMO}_{L}(X)$. When $X=\boldsymbol{R}^{n}$ and $L$ is a divergence form elliptic operator, the authors in [20] proved that the dual space of the Hardy space associated to $L$ is the space of VMO type associated to $L$. In this section we will study the duality between $H_{L}(X)$ and $\mathrm{VMO}_{L}(X)$. More precisely, the main result in this section is formulated by the following theorem.

THEOREM 4.1. Assume that L satisfies $(\mathrm{H} 1)$ and $(\mathrm{H} 2)$ and $M>n / 4$. Then the dual space of $\mathrm{VMO}_{L}(X)$ is the space $H_{L}(X)$ in the following sense.

(a) Suppose $f \in H_{L}(X)$. Then the linear functional l given by

$$
l(g)=\int_{X} f(x) g(x) d \mu(x)
$$

initially defined on the dense subspace $\mathrm{VMO}_{L}(X) \cap L^{2}(X)$, has a unique extension to $\mathrm{VMO}_{L}(X)$.

(b) Conversely, every continuous linear functional l on $\mathrm{VMO}_{L}(X)$ can be realized uniquely as in (a), with $f \in H_{L}(X)$ and

$$
\|f\|_{H_{L}(X)} \leq c\|l\|_{\left(\mathrm{VMO}_{L}(X)\right)^{*}} .
$$

Before giving a proof of Theorem 4.1, we establish some technical lemmas. Let $M \in N$. For an $F \in L^{2}(X \times(0, \infty))$ with bounded support, define

$$
\pi_{L, M} F(x)=C_{M} \int_{0}^{\infty}\left(t^{2} L\right)^{M} e^{-t^{2} L} F(\cdot, t)(x) \frac{d t}{t},
$$

where $C_{M}$ is a constant satisfying $C_{M} \int_{0}^{\infty} t^{2(M+1)} e^{-2 t^{2}} \frac{d t}{t}=1$.

LEMMA 4.2. The operator $\pi_{L, M}$ initially defined on $T_{2, b}^{p}$ extends to a bounded linear operator:

(a) from $T_{2}^{p}$ to $L^{p}$ if $1<p<\infty$;

(b) from $T_{2}^{1}$ to $H_{L}(X)$;

(c) from $T_{2}^{\infty}$ to $\mathrm{BMO}_{L}(X)$;

(d) from $T_{2, V}^{\infty}$ to $\mathrm{VMO}_{L}(X)$.

PROOF. The proof of (a) and (b) can be found in [18, Proposition 9.3]. The proof of (c) is analogous to one of [14, Lemma 4.3 (c)]. To prove (d), assume that $f \in T_{2, V}^{\infty}$. We will claim that $\pi_{L, M} f \in \mathrm{VMO}_{L}(X)$. By Proposition 3.16, it is equivalent to that $\left(t^{2} L\right)^{K} e^{-t^{2} L_{\pi}} \pi_{L, M} f \in$ $T_{2, V}^{\infty}$ for $K>M+n / 4$. Note that since $T_{2, b}^{2}$ is dense in $T_{2, V}^{\infty}$, we need only to treat functions $f \in T_{2, b}^{2}$. By an argument similar to that above, it suffices to show that

$$
\left(\frac{1}{V(B)} \int_{\widehat{B}}\left|\left(t^{2} L\right)^{K} e^{-t^{2} L} \pi_{L, M} f\right|^{2} d \mu(x) \frac{d t}{t}\right)^{1 / 2} \leq c \sum_{j=2}^{\infty} 2^{-2 j(K-n / 4)} w_{j}(f, B),
$$

where

$$
w_{k}(f, B)=\left(\frac{1}{V\left(2^{k} B\right)} \int_{\widehat{2^{k} B}}|f(x, t)|^{2} d \mu(x) \frac{d t}{t}\right)^{1 / 2} .
$$


To do this, split $f$ into $f:=f \chi_{\widehat{4 B}}+\sum_{j=3}^{\infty} f \chi_{V_{j}(B)}$, where $V_{j}(B)=\widehat{2^{j} B} \widehat{2^{j-1} B}$. We then have

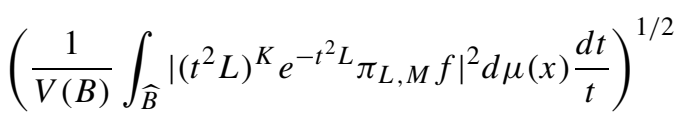

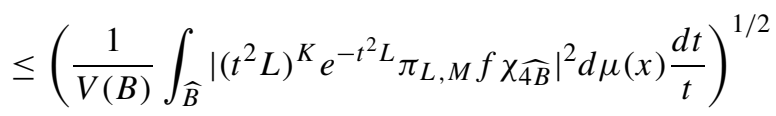

$$
\begin{aligned}
& +\sum_{j=3}^{\infty}\left(\frac{1}{V(B)} \int_{\widehat{B}} \mid\left(t^{2} L\right)^{K} e^{\left.-\left.t^{2} L_{\pi, M} f \chi_{V_{j}(B)}\right|^{2} d \mu(x) \frac{d t}{t}\right)^{1 / 2}}\right. \\
& \leq \boldsymbol{A}+\sum_{j=3}^{\infty} \boldsymbol{B}_{j}
\end{aligned}
$$

For the term $\boldsymbol{A}$, we have

$$
\begin{aligned}
\boldsymbol{A} & \leq \frac{1}{V(B)}\left\|\pi_{L, M} f \chi_{\widehat{4 B}}\right\|_{L^{2}(X)} \\
& \leq \frac{1}{V(4 B)}\left\|f \chi_{4 B_{B}}\right\|_{T_{2}^{2}} \quad \text { due to (a) } \\
& =c w_{2}(f, B) .
\end{aligned}
$$

For $j \geq 3$, we have, by Minkowski's inequality,

$$
\begin{aligned}
& V(B)^{1 / 2} \boldsymbol{B}_{j} \\
& =\left(\int_{\widehat{B}}\left|\int_{2^{j-1} r_{B}}^{2^{j} r_{B}}\left(t^{2} L\right)^{K} e^{-t^{2} L}\left(s^{2} L\right)^{M} e^{-s^{2} L} f(\cdot, s) \chi_{S_{j}(B)} \frac{d s}{s}\right|^{2} d \mu(x) \frac{d t}{t}\right)^{1 / 2} \\
& \leq \int_{2^{j-1} r_{B}}^{2^{j} r_{B}}\left(\int_{\widehat{B}}\left|\frac{t^{2 K} s^{2 M}}{\left(t^{2}+s^{2}\right)^{K+M}}\left(\left(t^{2}+s^{2}\right) L\right)^{K+M} e^{-\left(t^{2}+s^{2}\right) L} f(\cdot, s) \chi_{S_{j}(B)}\right|^{2} d \mu(x) \frac{d t}{t}\right)^{1 / 2} \frac{d s}{s} \\
& \leq C \int_{2^{j-1} r_{B}}^{2^{j} r_{B}}\left(\int_{\widehat{B}} \mid \frac{t^{4 K} s^{4 M}}{\left(t^{2}+s^{2}\right)^{2 K+2 M}}\left[\frac{\left(t^{2}+s^{2}\right)}{\left(2^{j} r_{B}\right)^{2}}\right]^{2 K+2 M}\left\|f(\cdot, s) \chi_{S_{j}(B)}\right\|_{L^{2}(X)}^{2} \frac{d t}{t}\right)^{1 / 2} \frac{d s}{s} \\
& \leq C \int_{2^{j-1} r_{B}}^{2^{j} r_{B}} \frac{r_{B}^{2 K}\left(2^{j} r_{B}\right)^{2 M}}{\left(2^{j} r_{B}\right)^{2(K+M)}\left\|f(\cdot, s) \chi_{S_{j}(B)}\right\|_{L^{2}(X)} \frac{d s}{s}} \\
& \leq C 2^{-2 j K} \int_{2^{j-1} r_{B}}^{2^{j} r_{B}}\left\|f(\cdot, s) \chi_{S_{j}(B)}\right\|_{L^{2}(X)} \frac{d s}{s} \\
& \leq C 2^{-2 j K+j \frac{n}{2}} V(B)^{1 / 2} w_{j}(f, B),
\end{aligned}
$$

where in the last inequality we apply Hölder's inequality. This implies

$$
\boldsymbol{B}_{j} \leq C 2^{-2 j(K-n / 4)} w_{j}(f, B) .
$$


The some ideas in following lemma are inspired by [20]. However, we need some improvements to adapt to our settings.

LEMMA 4.3. $\operatorname{VMO}_{L}(X) \cap L^{2}(X)$ is dense in $\operatorname{VMO}_{L}(X)$.

Proof. For any $f \in \mathrm{VMO}_{L}(X)$ and $M>n / 4$. By Proposition 3.16, $g:=$ $\left(t^{2} L\right)^{M} e^{-t^{2} L} f \in T_{2, V}^{\infty}$. By the density of $T_{2, b}^{2}$ in $T_{2, V}^{\infty}$, we can pick a sequence $\left\{g_{k}\right\}$ in $T_{2, b}^{2}$

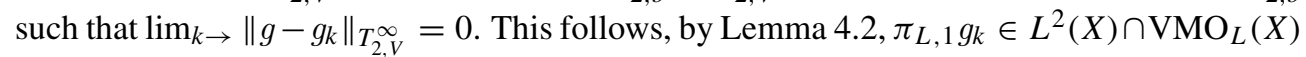

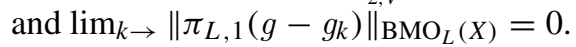

Let $\alpha$ be a $(1,2, M)$-atom in $H_{L}(X)$, by the definition of $H_{L}(X), t^{2} L e^{-t^{2} L} \alpha \in T_{2}^{1}$. On the other hand, $\left(H_{L}(X)\right)^{*}=\mathrm{BMO}_{L}(X)$, see [18], and $\left(T_{2}^{1}\right)^{*}=T_{2}^{\infty}$. Therefore, we have by [19, Corollary 4.2]

$$
\begin{aligned}
\int_{X} f(x) \alpha(x) d \mu(x) & =C_{M} \int_{X \times(0, \infty)}\left(t^{2} L\right)^{M} e^{-t^{2} L} f(x) t^{2} L e^{-t^{2} L} \alpha(x) d \mu(x) \frac{d t}{t} \\
& =C_{M} \lim _{k \rightarrow \infty} \int_{X \times(0, \infty)} g_{k}(x) t^{2} L e^{-t^{2} L} \alpha(x) d \mu(x) \frac{d t}{t} \\
& =C \lim _{k \rightarrow \infty} \int_{X} \pi_{L, 1} g_{k}(x) \alpha(x) d \mu(x) \\
& =C \lim _{k \rightarrow \infty}\left\langle\pi_{L, 1} g_{k}, \alpha\right\rangle .
\end{aligned}
$$

Since the subspace of all finite linear combination of atoms is dense in $H_{L}(X), \| \pi_{L, 1} g_{k}-$ $f\left\|_{\mathrm{VMO}_{L}(X)}=\right\| \pi_{L, 1} g_{k}-f\left\|_{\mathrm{BMO}_{L}(X)}=\right\| \pi_{L, 1} g_{k}-f \|_{\left(H_{L}(X)\right)^{*}} \rightarrow 0$.

We are now in position to prove the main result.

Proof of Theorem 4.1. (a) Since $\operatorname{VMO}_{L}(X) \subset \mathrm{BMO}_{L}(X)=\left(H_{L}(X)\right)^{*}, H_{L}(X) \subset$ $\left(H_{L}(X)\right)^{* *} \subset\left(\mathrm{VMO}_{L}(X)\right)^{*}$.

(b) We adapt some ideas in [14] to our present situation. For $M$ sufficiently large, define

$$
\Omega_{L}=\left\{h ; h=\left(t^{2} L\right)^{M} e^{-t^{2} L} g \text { for some } g \in \operatorname{VMO}_{L}(X)\right\} .
$$

By Proposition 3.16, $\Omega_{L} \subset T_{2, V}^{\infty}$. Moreover, for $h \in T_{2, V}^{\infty}$, Lemma 4.2 shows that $\pi_{L, M} h \in$ $\operatorname{VMO}_{L}(X)$. Therefore, for any $l \in\left(\operatorname{VMO}_{L}(X)\right)^{*}$ and $g \in \operatorname{VMO}_{L}(X) \cap L^{2}(X)$, we have

$$
l(g)=l \circ \pi_{L, M} \circ\left(t^{2} L\right)^{M} e^{-t^{2} L} g .
$$

We can prove that $l \circ \pi_{L, M}$ is a continuous linear functional on $\Omega_{L}$ and satisfies

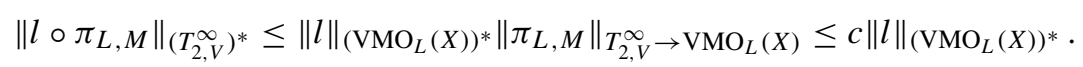

By the Hahn-Banach theorem, $l \circ \pi_{L, M}$ can be extended to a continuous linear functional on $T_{2, V}^{\infty}$. Since $\left(T_{2, V}^{\infty}\right)^{*}=T_{2}^{1}$, there exists a $w(x, t) \in T_{2}^{1}$ with $\|w\|_{T_{2}^{1}} \leq c\left\|l \circ \pi_{L, M}\right\|$ such that

$$
\begin{aligned}
l(g) & =l \circ \pi_{L, M} \circ\left(t^{2} L\right)^{M} e^{-t^{2} L} g \\
& =\int_{X \times(0, \infty)} w(x, t)\left(t^{2} L\right)^{M} e^{-t^{2} L} g(x) d \mu(x) \frac{d t}{t}
\end{aligned}
$$




$$
\begin{aligned}
& =\int_{X} g(x)\left(\int_{0}^{\infty}\left(t^{2} L\right)^{M} e^{-t^{2} L} w(\cdot, t)(x) \frac{d t}{t}\right) d \mu(x) \\
& =\int_{X} g(x) \pi_{L, M} w(x) d \mu(x) .
\end{aligned}
$$

According to Lemma 4.2, $\pi_{L, M} w \in H_{L}(X)$ and $\left\|\pi_{L, M} w\right\| \leq c\|w\|_{T_{2}^{1}} \leq c\left\|l \circ \pi_{L, M}\right\| \leq c\|l\|$.

Acknowledgements. The author is partly supported by NAFOSTED under the project "Some optimization and control problems for dynamical systems under uncertainties". He would like to thank the supervisor, Prof. X. T. Duong, for helpful comments and suggestions. He also thanks the editor for his useful comments to improve the paper.

\section{REFERENCES}

[ 1] P. AUSCHER, On necessary and sufficient conditions for $L^{p}$-estimates of Riesz transforms associated to elliptic operators on $\boldsymbol{R}^{n}$ and related estimates, Mem. Amer. Math. Soc. 186 (2007), no. 871, xviii+75 pp.

[2] P. Auscher, X. T. Duong And A. McIntosh, Boundedness of Banach space valued singular integral operators and Hardy spaces, Unpublished preprint, 2005.

[ 3 ] P. Auscher, T. Coulhon, X. T. Duong And S. Hofmann, Riesz transform on manifolds and heat kernel regularity, Ann. Sci. École Norm. Sup. (4) 37 (2004), 911-957.

[ 4 ] P. Auscher, A. McIntosh And E. Russ, Hardy spaces of differential forms on Riemannian manifolds, J. Geom. Anal. 18 (2008), 192-248.

[ 5 ] P. AusCher AND E. RUSS, Hardy spaces and divergence operators on strongly Lipschitz domain of $\boldsymbol{R}^{n}, \mathrm{~J}$. Funct. Anal. 201 (2003), 148-184.

[6] P. Auscher And P. Tchamitchian, Square root problem for divergence operators and related topics, Astérisque 249, Soc. Math. France (1998), viii+172 pp.

[ 7 ] M. Christ, A $T(b)$ theorem with remarks on analytic capacity and the Cauchy integral, Colloq. Math. 60/61 (1990), 601-628.

[ 8 ] T. Coulhon And X. T. Duong, Maximal regularity and kernel bounds: observations on a theorem by Hieber and Prüss, Adv. Differential Equations 5 (2000), 343-368.

[9] R. R. Coifman, Y. Meyer and E. M. Stein, Some new functions and their applications to harmonic analysis, J. Funct. Anal. 62 (1985), 304-335.

[10] R. R. Coifman, Y. Meyer and E. M. Stein, Un nouvel espace adapté a l'étude des opérateurs définis par des itégral singuliéres, Harmonic analysis (Cortoona, 1982), Lecture Notes in Math. 992, 1-15, SpringerVelarg, Berlin, 1983.

[11] R. R. Coifman And G. Weiss, Extension of hardy spaces and their use in analysis, Bull. Amer. Math. Soc. 83 (1977), 569-645.

[12] D. Deng, X. T. Duong, L. Song, C. TAN And L. Yan, Functions of vanishing mean oscillation associated with operators and applications, Michigan Math. J. 56 (2008), 529-550.

[13] X. T. DUONG AND L. X. YAn, New function spaces of BMO type, the John-Nirenberg inequality, interpolation and applications, Comm. Pure Appl. Math. 58 (2005), 1375-420.

[14] X. T. DuOng AND L. X. YAn, Duality of Hardy and BMO spaces associated with operators with heat kernel bounds, J. Amer. Math. Soc. 18(2005), 943-973.

[15] C. Fefferman And E. M. Stein, $H^{p}$ spaces of several variables, Acta Math. 129 (1972), 137-193.

[16] S. Hofmann AND J. M. Martell, $L^{p}$ bounds for Riesz transforms and square roots associated to second order elliptic operators, Publ. Mat. 47 (2003), 497-515. 
[17] S. HofmAnn AND S. MAYBoroda, Hardy and BMO spaces associated to divergence form elliptic operators, Math. Ann. 344 (2009), 37-116.

[18] S. Hofmann, G. Lu, D. Mitrea, M. Mitrea And L. Yan, Hardy spaces associated to non-negative selfadjoint operators satisfying Davies-Gaffney estimates, Mem. Amer. Math. Soc. 214 (2011), no. 1007, vi+78 pp.

[19] R. JIANG AND D. YANG, New Orlicz-Hardy spaces associated with operators satisfying Davies-Gaffney estimates, Commun. Contemp. Math. 13 (2011), 331-373.

[20] R. JIANG AND D. YANG, Generalized vanishing mean oscillation spaces associated with divergence form elliptic operators, Integral Equations Operator Theory 67 (2010), 123-149.

[21] F. John And L. Nirenberg, On functions of bounded mean osillation, Comm. Pure. Appl. Math. 14 (1961), 415-426.

[22] E. RUSS, The atomic decomposition for tent spaces on spaces of homogeneous type, Asymptotic Geometric Analysis, Harmonic Analysis, and Related Topics, 125-135, Proceedings of the Centre for Mathematical Analysis, Australian National University, 42, Australian National University, Canberra, 2007.

[23] D. SARAson, Functions of vanishing mean oscillation, Trans. Amer. Math. Soc. 207 (1975), 391-405.

[24] W. WANG, The predual spaces of tent spaces and some characterizations of $\lambda_{\alpha}\left(\boldsymbol{R}^{n}\right)$ spaces, Beijing Daxue Xuebao 24 (1988), 535-551.

DePartMENT OF MATHEMATICS

MACQUARIE UNIVERSITY

SYDNEY 2109 NSW

Australia

E-mail address: the.bui@mq.edu.au 